\title{
Mean First Passage Time of Preferential Random Walks on Complex Networks with Applications
}

\author{
Zhongtuan Zheng, ${ }^{1}$ Gaoxi Xiao, ${ }^{2}$ Guoqiang Wang, ${ }^{1}$ Guanglin Zhang, ${ }^{3}$ and Kaizhong Jiang ${ }^{1}$ \\ ${ }^{1}$ School of Mathematics, Physics and Statistics, Shanghai University of Engineering Science, Shanghai 201620, China \\ ${ }^{2}$ School of Electrical and Electronic Engineering, Nanyang Technological University, Singapore 639798 \\ ${ }^{3}$ School of Information Science and Technology, Donghua University, Shanghai 201620, China
}

Correspondence should be addressed to Zhongtuan Zheng; zhongtuanzheng@163.com

Received 23 March 2017; Accepted 14 May 2017; Published 17 August 2017

Academic Editor: Huanqing Wang

Copyright (C) 2017 Zhongtuan Zheng et al. This is an open access article distributed under the Creative Commons Attribution License, which permits unrestricted use, distribution, and reproduction in any medium, provided the original work is properly cited.

\begin{abstract}
This paper investigates, both theoretically and numerically, preferential random walks (PRW) on weighted complex networks. By using two different analytical methods, two exact expressions are derived for the mean first passage time (MFPT) between two nodes. On one hand, the MFPT is got explicitly in terms of the eigenvalues and eigenvectors of a matrix associated with the transition matrix of PRW. On the other hand, the center-product-degree (CPD) is introduced as one measure of node strength and it plays a main role in determining the scaling of the MFPT for the PRW. Comparative studies are also performed on PRW and simple random walks (SRW). Numerical simulations of random walks on paradigmatic network models confirm analytical predictions and deepen discussions in different aspects. The work may provide a comprehensive approach for exploring random walks on complex networks, especially biased random walks, which may also help to better understand and tackle some practical problems such as search and routing on networks.
\end{abstract}

\section{Introduction}

In the past two decades, as the effective modelling of a wide range of complex systems, complex networks have attracted much attention from both theorists and technologists [1]. Most efforts were devoted to uncover the universal topological properties of real systems [2]. Many empirical studies revealed that a large variety of real-world networks display simultaneously small-world phenomenon [3] and scale-free nature [4]. These global properties imply a large connectivity heterogeneity. It is evidenced by power-law degree distributions and small average distance between nodes, together with strong clustering. But an even more intriguing task is to understand the interplay between the structure of complex networks and various dynamical processes taking place on them. The processes include epidemic spreading [5] and traffic flow [6]. Such processes have potential applications in the control of stochastic systems [7-10]. It has been demonstrated that the structural properties of networks play an important role in determining the dynamical features of these processes [2].

As a paradigmatic dynamical process, random walks on complex networks [11] have been widely explored due to their basic dynamic properties and broad applications [12]. In recent years, there has been increasing interest in random walks on small-world networks $[13,14]$ and scalefree networks $[15,16]$. The structural properties affect deeply the nature of the diffusive and relaxation dynamics of the random walk $[14,16]$. Such interest is well motivated since the random walks could also be a mechanism of search and routing on complex networks [17-21]. Random walks can be used to detect unknown paths [20], design dynamic routing in wireless sensor networks [21], and so on. Furthermore, to improve search performance, various modified random walks schemes have been proposed, such as self-avoiding walks [22] and coverage-adaptive walks [23].

Those modified random-walk strategies, however, are in most cases too complicated to be solved analytically. 
In addition, despite some studies of biased or preferential random walks [18, 24-26], a general framework for the scaling behaviour of the walks in networks with different topologies has not been available. That is to say, there is not a unified approach for understanding the behaviour of biased random walks. In this paper, we will develop a simplified random-walk model of unifying different randomwalk strategies so that one could better understand results about mean first passage time (MFPT).

MFPT is an important characteristic of random walks on networks, which is investigated in various situations, especially in characterizing search efficiency $[17,19,27]$. The MFPT from node $i$ to $j$, denoted by $\left\langle T_{i j}\right\rangle$, is the expected steps taken by a walker to reach node $j$ for the first time starting from node $i$. In complex networks, MFPTs of random walks heavily depend on the underlying network topology. MFPT of a single random walker in complex networks [28] has been extensively studied. For random walks on the family of small-world networks, mean field approximation was applied to get the analytic result for MFPT [13]. By using Laplace transform, an exact expression for the MFPT of random walks on complex networks was derived [11]. Adopting the theory proposed in [29] led to explicit solutions of the MFPT for random walks on self-similar networks [30]. The solutions highlighted two strongly different scaling behaviours of the MFPT for different types of random walks. For random walks in a general graph, an explicit formula of the global MFPT to a trap node was provided [31]. The formula is expressed in terms of eigenvalues and eigenvectors of Laplacian matrix for the graph.

However, those results about MFPT are in various forms and difficultly make a unified understanding. In many circumstances, they are not beneficial for revealing the interactions between the structural properties and randomwalk dynamical behaviours. Moreover, the impacts of node strength on scaling properties of the MFPT remain less understood. To meet the above shortfall, we will attempt to establish a unified random-walk model in a tractable way. And we expect that some unified analytic results could be obtained for the statistics of the random-walk system. For this object, we take advantage of random walks on weighted networks and thus can make use of reversible Markov chains theory. Based on local information of the degrees of current node and its nearest neighbors, we attach different edge weights and then construct different random walks on weighted networks. We focus on preferential random walks (PRW) and simple random walks (SRW). We can consider the influence of node strength on the behaviour of random walks by PRW and SRW.

In the following, we develop a comprehensive approach for exploring the scaling behaviour of discrete-time random walks on complex networks. We mainly investigate PRW on complex networks and make comparative study with SRW. In Section 2, we give preliminaries and terminologies for random walks. In Section 3, we first attach weight $c_{i j}=d_{j} d_{i}$ to each edge and construct PRW through random walks on weighted networks. Then, we derive two exact expressions of the MFPT between two nodes for PRW on networks. One is a spectra formula obtained by the method of matrix analysis; the other is a probabilistic formula got by the method of stopping time. Accordingly, based on the two formulas for MFPT, we get the analytical formulas of the average over MFPTs (AMFPTs) between all node pairs. In Section 4, numerical simulations of an ensemble of random walkers moving on paradigmatic network models confirm analytical predictions and deepen discussions in different aspects. The network models include simple ER random networks, NW small-world networks, and BA scale-free networks. We discuss the effects of the structural heterogeneity on the MFPT and AMFPT. Through the comparison of PRW and SRW in networks, we unveil the CPD-based assortativity of network structure. We also interpret and handle some searchrelated issues by random walks, such as search efficiency in target problem, sensitivity of the total average search cost affected by the source node's location, network searchability, and difference of the scaling behaviours for search cost among the three strategies of maximum-degree-search (MDS), PRW, and SRW.

\section{Preliminaries and Terminologies}

A simple random walk on a connected, undirected network $G$ with $N$ nodes is a Markov chain whose states are the nodes of $G$. The walk begins with a walker at some node, and at each tick of the clock, the walker moves to a neighbor of its current position at random (uniformly). If instead the transition probabilities are biased according to edge weights, one obtains a general reversible Markov chain. In this section, we give a brief introduction to reversible Markov chains and random walks on weighted networks. We review basic concepts and some fundamental issues that are handy in proving our main results.

We describe a discrete-time Markov chain as follows: Consider a stochastic process $\left(X_{t}: t=0,1,2, \ldots\right)$ with a finite state space $V=\{1,2, \ldots, N\}$. The process starts in one of these states and moves successively from one state to another. If the chain is currently in state $i_{n}$, then it moves to state $i_{n+1}$ at the next step with a probability denoted by $p_{i j}$, and this probability is independent of the past states and depends only on the current state; that is,

$$
\begin{aligned}
& P\left(X_{n+1}=i_{n+1} \mid X_{0}=i_{0}, X_{1}=i_{1}, \ldots, X_{n}=i_{n}\right) \\
& \quad=P\left(X_{n+1}=i_{n+1} \mid X_{n}=i_{n}\right)=p_{i j},
\end{aligned}
$$

where $i_{0}, i_{1}, \ldots, i_{n+1} \in V, n \geq 0$.

The probabilities $p_{i j}=P\left(X_{n+1}=j \mid X_{n}=i\right)$ are called one-step transition probabilities, which constitute the transition matrix $P=\left(p_{i j}\right)$ of the chain. Accordingly, the $t$ steps transition probabilities are $P\left(X_{t}=j \mid X_{0}=i\right)=p_{i j}^{(t)}$, where $P^{(t)}=P P \cdots P$ is the $t$-fold matrix product. Write $P_{i}(\cdot)$ and $E_{i}(\cdot)$ for probabilities and expectations for the chain starting at state $i$ and time 0 . More generally, write $P_{\rho}(\cdot)$ and $E_{\rho}(\cdot)$ for probabilities and expectations for the chain starting at time 0 with distribution $\rho$.

For the Markov chain with the state space $V=$ $\{1,2, \ldots, N\}$, we say that the distribution $\pi=\left(\pi_{1}, \pi_{2}, \ldots\right.$, $\left.\pi_{N}\right)^{\top}$ is stationary or steady for the state space $V$ if $\pi^{\top}=\pi^{\top} P$; 
that is, for any $j \in V, \pi_{j}=\sum_{i \in V} \pi_{i} p_{i j}$. It is well known that any finite irreducible aperiodic Markov chain has exactly one stationary distribution [32]. The stationary distribution plays the main role in asymptotic results as follows. We consider a finite irreducible Markov chain with the stationary distribution $\pi$. Let $N_{i}(t)$ be the number of visits to state $i$ during times $(0,1, \ldots, t-1)$. Then for any initial distribution [33],

$$
\frac{N_{i}(t)}{t} \longrightarrow \pi_{i} \text { a.s, } \quad \text { as } t \longrightarrow \infty \text {. }
$$

If the chain is aperiodic, then, for all $i \in V$ [34],

$$
P\left(X_{t}=i\right) \longrightarrow \pi_{i}, \quad \text { as } t \longrightarrow \infty \text {. }
$$

Further, in terms of the stationary distribution, it is easy to formulate the property of time reversibility $[32,33]$ : it is equivalent to saying that for every pair $i, j \in V$

$$
\pi_{i} p_{i j}=\pi_{j} p_{j i}
$$

That is, in a chain with time reversibility, we step as often from $i$ to $j$ as from $j$ to $i$. More vividly, given that a move of the chain runs forwards and the same move runs backwards, you cannot tell which is which. At this point, we call the chain reversible.

Now, we shift attention to random walks on weighted networks $[35,36]$. We consider a finite nonbipartite network (or graph) $G=(V, E)$ with $N$ nodes (or vertices, sites) and $M$ edges connecting them. Here, we consider only a connected network; that is, there is at least one path linking any two nodes on the network. The connectivity is represented by the adjacency matrix $A$ with entries $a_{i j}, i, j=1,2, \ldots, N$. $a_{i j}=1$ if there is an edge between nodes $i$ and $j$; otherwise $a_{i j}=0$. We also assume all $a_{i i}=0$ conventionally. That is to say, the network we consider has no multiple edges and has no self-loops. The degree $d_{i}$ of node $i$ is defined as the number of connected neighbors; that is, $d_{i}=\sum_{j=1}^{N} a_{i j}$. For the network $G=(V, E)$, if $a_{i j}=1$, we assign a positive weight $0<c_{i j}=c_{j i}<\infty$ to edge $(i, j)$; otherwise, if $a_{i j}=0$, namely, the edge $(i, j)$ is absent, we attach weight $c_{i j}=c_{j i}=0$. Writing $c$ for the function $i j \mapsto c_{i j}$, we have obtained the weighted network $(G, c)[1,37]$.

We define a random walk on the weighted network as a sequence of random variables $\left(X_{t}: t=0,1,2, \ldots\right)$, each taking values in the set $V$ of nodes. And the walk is such that if $X_{t}=i$, namely, at time $t$ the walker is at node $i$, then with the transition probability $p_{i j}=c_{i j} / \sum_{j} c_{i j}$ the walker hops to one neighbor $j$ at the next time $t+1$; that is to say, the walker randomly selects a neighboring node as its next dwelling point according to edge weights. Clearly, the walk $\left(X_{t}: t=0,1,2, \ldots\right)$ can be described by a Markov chain with the finite space $V$, whose transition matrix $P$ satisfies $[35,36]$

$$
p_{i j}=P\left(X_{t+1}=j \mid X_{t}=i\right)= \begin{cases}\frac{c_{i j}}{c_{i}}, & (i, j) \in E, \\ 0, & (i, j) \notin E,\end{cases}
$$

where $c_{i}=\sum_{j \in \tau(i)} c_{i j}$. The sum $c_{i}$, called the strength of node $i$, runs over the set $\tau(i)$ of all the connected neighbors of $i$. Such a chain is reversible with the stationary distribution $[35,36]$

$$
\pi=\left(\pi_{1}, \pi_{2}, \ldots, \pi_{N}\right)^{\top}, \quad \text { where } \pi_{i}=\frac{c_{i}}{c},
$$

since $\pi_{i} p_{i j}=\pi_{j} p_{j i}=c_{i j} / c$. Note that $c=\sum_{i} c_{i}$ is the total edge weight, when each edge is counted twice, that is, once in each direction.

In fact, by configuring the edge weights $c_{i j}$, we can get corresponding node strengths $c_{i}$ [37] and thus can control the scaling behaviour of the random walks. The weight heterogeneity could play an essential role in dynamical processes on networks [6], including random-walk dynamics. This may also have potential reference value in the control design for stochastic systems [38-41]. If we assign weight $c_{i j}=1$ to each edge $(i, j)$, then the random walk on the weighted network is a simple random walk. The transition matrix of the simple random walk is described by

$$
p_{i j}= \begin{cases}\frac{1}{d_{i}}, & (i, j) \in E, \\ 0, & (i, j) \notin E .\end{cases}
$$

By using (6), it is easy to prove that the unique stationary distribution of the simple random walk becomes

$$
\pi=\left(\frac{d_{1}}{2 M}, \frac{d_{2}}{2 M}, \ldots, \frac{d_{N}}{2 M}\right)^{\top},
$$

where $M$ is the number of edges of the network $G$.

\section{Mean First Passage Time of Preferential Random Walks}

In this section, we present a systematic study of preferential random walks in a general connected nonbipartite network $G=(V, E)$ with $N$ nodes and $M$ edges. MFPT is one basic characteristic of the random walks, since it contains a great deal of useful knowledge about the random-walk dynamics. We will derive two analytical expressions for MFPT between source node and target node, based on which we obtain the closed-form formulas of AMFPT between all node pairs. First, through applying the matrix analysis approach proposed in $[42,43]$, we obtain an exact solution to the MFPT, which is expressed in terms of the eigenvalues and eigenvectors of a matrix associated with the transition matrix of PRW. Then, by employing the stopping time technique developed in [44], we get a probabilistic formula for the MFPT, which provides the dependence of MFPT on the CPD of target node.

3.1. Formulation of $P R W$. To perform a random walk on a complex network, each node needs to calculate the transition probability from the node to each of its neighbors, but the knowledge available to this endpoint is limited to its local information. Thus the real question we need to ask is: what is the local information necessary and sufficient to calculate good transition probabilities at each node? In this 
paper, we implement preferential random walks on complex networks, in which the walker is prone to a high-degree neighboring node. Preferential random walks on complex networks are defined by following rule: Suppose a particle (or random walker) wanders on the network. It randomly selects a neighboring node as its next dwelling point according to the degrees of neighboring nodes. That is to say, the probability of heading to any neighboring node is $p_{i j}=d_{j} / \sum_{j \in \tau(i)} d_{j}$, where $d_{j}$ denotes the degree, the number of connected neighbors, of a node $j$, and $\tau(i)$ denotes all the connected neighbors of node $i$. Representing $p_{i j}$ as $d_{j} d_{i} / \sum_{j \in \tau(i)} d_{j} d_{i}$, we can apply random walks on weighted networks to study preferential random walks and simple random walks as well. Thus, we can use a unified approach to explore preferential random walks and simple random walks.

If we attach weight $c_{i j}=d_{j} d_{i}$ to each edge $(i, j)$, then the random walk on the weighted network is a preferential random walk with the transition matrix as follows:

$$
p_{i j}= \begin{cases}\frac{d_{j} d_{i}}{\sum_{j \in \tau(i)} d_{j} d_{i}}, & (i, j) \in E, \\ 0, & (i, j) \notin E .\end{cases}
$$

According to (6), the preferential random walk has a stationary distribution $\pi=\left(\pi_{1}, \pi_{2}, \ldots, \pi_{N}\right)^{\top}$ that is a unique probabilistic vector satisfying

$$
\pi_{j}=\frac{\sum_{i \in \tau(j)}\left(d_{i} d_{j}\right)}{\sum_{j} \sum_{i \in \tau(j)}\left(d_{i} d_{j}\right)} .
$$

There is a measure of node strength, that is, $d_{j} \sum_{i \in \tau(j)} d_{i}$, in the definition of the PRW and the expression for the MFPT; see (9) and (43). We call it the center-product-degree (CPD) of the node $j$ and denote it by $\mathrm{CPD}_{j}$. The CPD heavily characterizes the behaviour of PRW on the network. There is a close relationship between CPD and network assortativity [1]. For a degree-correlation network, if the center-productdegree $\mathrm{CPD}_{j}$ of node $j$ is an increasing function of the degree $d_{j}$ of node $j$, then we say that the network is weekly assortative, whereas if the $\mathrm{CPD}_{j}$ is decreasing function of $d_{j}$, the network is strongly disassortative. Obviously, if the network is assortative, then it will be weakly assortative, while if the network is strongly disassortative, then it will be disassortative. We will numerically explore the CPDbased assortativity and homogeneity of network structure by random walks in Section 4.1.2.

In fact, the above-mentioned various types of biased random walks in networks [24-26] can also be transformed into random walks on weighted networks equivalently in similar way. For example, a biased random walk in uncorrelated networks and a biased $N$ lions-lamb model were introduced in $[24,25]$, respectively. In the two articles, the bias is defined by the preferential transition probability $p_{i j}=d_{j}^{\alpha} / \sum_{j \in \tau(i)} d_{j}^{\alpha}$, where $d_{j}$ denotes the degree of a node $j$ and $\tau(i)$ represents the set of node $i$ 's nearest neighbors. We can attach edge weight $c_{i j}=\left(d_{j} d_{i}\right)^{\alpha}$ and thus revisit the biased random walks. Another example is the Lévy random walks in [26] which can be got by configuring general weight $c_{i j}=\left(d_{i j}\right)^{-\alpha}$ between node $i$ and node $j$ where $d_{i j}$ denotes the shortest path length.
Remark 1. The framework here, together with the following main results, may provide a unified approach to improve the understanding of the behaviour of various random walks in networks, especially biased random walks.

3.2. Main Results. For the sake of clearness, let us first remind the reader of basic notions and terms about the MFPT. For node $j$, define two first passage times as

$$
\begin{aligned}
T_{j} & =\min \left\{t \geq 0: X_{t}=j\right\}, \\
T_{j}^{+} & =\min \left\{t \geq 1: X_{t}=j\right\} .
\end{aligned}
$$

As the random walks frequently start out at different initial nodes, it is important to distinguish the two first passage times. Write $\left\langle T_{i j}\right\rangle=E_{i} T_{j}^{+}$and $\left\langle T_{i j}^{\prime}\right\rangle=E_{i} T_{j}$, the angle bracket " \langle\rangle " represents "Mean." Given that $X_{0}=i$, of course $T_{j}=0$ when $j=i$; in this case we call $T_{j}^{+}$the first return time to node i. Correspondingly,

$$
\left\langle T_{i i}^{\prime}\right\rangle=E_{i} T_{i}=0,
$$

and we call

$$
\left\langle T_{i i}\right\rangle=E_{i} T_{i}^{+}
$$

the mean first return time (MFRT) to node $i$, that is, the mean number of steps needed to return to any starting point $i$. On the other hand, if $i \neq j$,

$$
\left\langle T_{i j}^{\prime}\right\rangle=E_{i} T_{j}=E_{i} T_{j}^{+}=\left\langle T_{i j}\right\rangle ;
$$

in this situation we call them the mean first passage time (MFPT) of $j$ from $i$, namely, the expected time it takes to reach node $j$ starting from node $i$. Occasionally, $\left\langle T_{i j}\right\rangle=E_{i} T_{j}^{+}$is also called the mean access time or the mean hitting time of $j$ from $i$.

3.2.1. Method of Matrix Analysis. We now extend the matrix analysis approach developed in $[42,43]$ to compute the MFPT $\left\langle T_{i j}\right\rangle(i \neq j)$ of a discrete-PRW walker to target node $j$ and the AMFPT. We thus get explicitly their dependence on the eigenvalues and eigenvectors of a matrix associated with the transition matrix of the PRW. We finish the calculation and derivation in the following two steps.

(i) Diagonalizing the transition matrix $P$ of the PRW

We use $P$ to define one matrix

$$
S: s_{i j}=\pi_{i}^{1 / 2} p_{i j} \pi_{j}^{-1 / 2}, \quad \text { namely } S=D_{\pi}^{-1 / 2} P D_{\pi}^{1 / 2},
$$

where $D_{\pi}=\operatorname{diag}\left(1 / \pi_{j}\right)$ is a diagonal matrix, of which $\pi_{j}=$ $\sum_{i \in \mathcal{\tau}(j)}\left(d_{i} d_{j}\right) / \sum_{j} \sum_{i \in \tau(j)}\left(d_{i} d_{j}\right), j=1,2, \ldots, N$.

Clearly, $S$ is symmetric due to the time reversibility of the PRW; namely, $\pi_{i} p_{i j}=\pi_{j} p_{j i}$. Then $S$ can be diagonalized and has the same set of eigenvalues as $P$. Let $\lambda_{1}, \lambda_{2}, \ldots, \lambda_{N}$ be the $N$ eigenvalues of $S$, rearranged as $1=\lambda_{1}>\lambda_{2} \geq$ $\cdots \geq \lambda_{N} \geq-1$, and let $u_{1}, u_{2}, \ldots, u_{N}$ be the corresponding orthogonal eigenvectors of unit length. Here we take $u_{1}=$ 
$\left(\pi_{1}^{1 / 2}, \pi_{2}{ }^{1 / 2}, \ldots, \pi_{N}^{1 / 2}\right)^{T}$ since the relation $\sum_{i} \pi_{i} p_{i j}=\pi_{j}$ holds, $j=1,2, \ldots, N$. Hence we can describe $S$ in a spectral representation:

$$
S=U \Lambda U^{T}=\sum_{k=1}^{N} \lambda_{k} u_{k} u_{k}^{T}
$$

Considering (15) and (16), one can easily obtain

$$
P=P^{*}+\sum_{k=2}^{N} \lambda_{k} D_{\pi}^{1 / 2} u_{k} u_{k}^{T} D_{\pi}^{-1 / 2},
$$

where $P^{*}=1 \pi^{T}$; that is, the entry of $P^{*}$ is $p_{i j}^{*}=\pi_{j}$.

(ii) Constituting matrix $T^{\prime}$ with MFPTs $\left\langle T_{i j}^{\prime}\right\rangle=E_{i} T_{j}$ and solving the matrix equation for $T^{\prime}$

Since the first step takes the walker to a neighbor $v$ of node $i$ with the probability $p_{i v}=d_{v} d_{i} / \sum_{v} d_{v} d_{i}$, one has

$$
\left\langle T_{i j}^{\prime}\right\rangle=1+\frac{d_{v} d_{i}}{\sum_{v} d_{v} d_{i}} \sum_{v \in \tau(i)}\left\langle T_{v j}^{\prime}\right\rangle,
$$

if $i \neq j$. According to (18), we can write an expression in matrix notation

$$
F=J+P T^{\prime}-T^{\prime}
$$

where any element of matrix $J$ is 1 . Applying (18) says that

$$
F_{i j}=0 \text {, }
$$

and hence $F$ is a diagonal matrix. The definition of the stationary distribution for the PRW indicates that $(P-I)^{T} \pi=$ 0 . Thus, $F^{T} \pi=J \pi+T^{\prime T}(P-I) \pi=J \pi=1$. That is to say,

$$
(F)_{i i}=\frac{1}{\pi_{i}}=\frac{\sum_{i} \sum_{j \in \tau(i)}\left(d_{i} d_{j}\right)}{\sum_{j \in \tau(i)}\left(d_{i} d_{j}\right)} .
$$

From (19)-(21), one immediately sees the matrix $T^{\prime}$ satisfies

$$
(I-P) T^{\prime}=J-D_{\pi}
$$

We will next solve this matrix equation for $T^{\prime}$. Unfortunately, (22) cannot uniquely determine $T^{\prime}$ since $I-P$ does not have an inverse. But following [43], $\left(I-P+P^{*}\right)^{-1}$ exists and

$$
\begin{aligned}
T^{\prime} & =\left(I-P+P^{*}\right)^{-1}\left(J-D_{\pi}\right)+P^{*} T^{\prime} \\
& =J-\left(I-P+P^{*}\right)^{-1} D_{\pi}+P^{*} T^{\prime}=H+P^{*} T^{\prime},
\end{aligned}
$$

where

$$
\begin{aligned}
H & =\left(I-P+P^{*}\right)^{-1}\left(J-D_{\pi}\right) \\
& =J-\left(I-P+P^{*}\right)^{-1} D_{\pi} .
\end{aligned}
$$

Note that $P^{*}=\mathbf{1} \pi^{T}$; from (23), one sees that

$$
\left\langle T_{i j}^{\prime}\right\rangle=H_{i j}+\left(\pi^{T} T^{\prime}\right)_{j} .
$$

Recalling that $\left\langle T_{i i}^{\prime}\right\rangle=0$ in (12) and from (23) one has

$$
0=H_{j j}+\left(\pi^{T} T^{\prime}\right)_{j} .
$$

From (25) and (26), we have

$$
\left\langle T_{i j}^{\prime}\right\rangle=H_{i j}-H_{j j}
$$

To give explicitly the spectra formula for the MFPT $\left\langle T_{i j}^{\prime}\right\rangle$, we will continue to do some calculation on $H$. Substituting (17) into (24), we obtain

$$
\begin{aligned}
H & =J-\left(I-P+P^{*}\right)^{-1} D_{\pi}=J-\left(D_{\pi}^{-1}\right. \\
& \left.-\sum_{k=2}^{N} \lambda_{k} D_{\pi}^{-1 / 2} u_{k} u_{k}^{T} D_{\pi}^{-1 / 2}\right)^{-1}=J-\left[D_{\pi}^{-1 / 2} U\right. \\
& \cdot \operatorname{diag}(1,1, \ldots, 1) U^{T} D_{\pi}^{-1 / 2}-D_{\pi}^{-1 / 2} U \\
& \left.\cdot \operatorname{diag}\left(0, \lambda_{2}, \ldots, \lambda_{N}\right) U^{T} D_{\pi}^{-1 / 2}\right]^{-1}=J-\left[D_{\pi}^{-1 / 2} U\right. \\
& \left.\cdot \operatorname{diag}\left(1,1-\lambda_{2}, \ldots, 1-\lambda_{N}\right) U^{T} D_{\pi}^{-1 / 2}\right]^{-1}=J \\
& -D_{\pi}^{1 / 2} U \operatorname{diag}\left(1, \frac{1}{1-\lambda_{2}}, \ldots, \frac{1}{1-\lambda_{N}}\right) U^{T} D_{\pi}^{1 / 2} .
\end{aligned}
$$

Rewriting the entries $H_{i j}$ and $H_{j j}$ of $H$ in (28) and plugging them into (27), we immediately get the following formula.

$$
\left\langle T_{i j}\right\rangle=\left\langle T_{i j}^{\prime}\right\rangle=\sum_{k=2}^{N} \frac{1}{1-\lambda_{k}}\left(\frac{u_{k j}^{2}}{\pi_{j}}-\frac{u_{k i} u_{k j}}{\sqrt{\pi_{j} \pi_{i}}}\right)
$$

$(i \neq j)$.

Taking into account (45), together with (29), we can calculate the average over MFPTs (AMFPT) between all node pairs $i j(i \neq j)$ as follows.

$$
\begin{aligned}
\left\langle T_{i j}\right\rangle & =\sum_{i} \sum_{j} \pi_{i} \pi_{j}\left\langle T_{i j}\right\rangle=\sum_{j} \pi_{j}\left\langle T_{i j}\right\rangle \\
& =\sum_{j} \sum_{k=2}^{N} \frac{1}{1-\lambda_{k}}\left(u_{k j}^{2}-u_{k i} u_{k j} \sqrt{\frac{\pi_{j}}{\pi_{i}}}\right) \\
& =\sum_{k=2}^{N} \frac{1}{1-\lambda_{k}}\left(\sum_{j} u_{k j}^{2}-u_{k i} \sqrt{\frac{1}{\pi_{i}}} \sum_{j} u_{k j} \sqrt{\pi_{j}}\right) \\
& =\sum_{k=2}^{N} \frac{1}{1-\lambda_{k}},
\end{aligned}
$$

where (16) has been used.

Remark 2. Summing up the above equations and derivation, (29) and (30) are our one central result for the MFPT $\left\langle T_{i j}\right\rangle(i \neq j)$ and AMFPT $\left\langle T_{i j}\right\rangle$, which are expressed in terms of the eigenvalues and eigenvectors of $S$ related to the transition matrix $P$ of the PRW. 
Remark 3. For SRW on the finite network, a similar result of (29) and (30) can be obtained from similar derivation above. The transition matrices of PRW and SRW, as two stochastic matrices, have similar spectral property [45]. Combining with (30), this indicates that the AMFPTs $\left\langle T_{i j}\right\rangle$ s of PRW and SRW have similar scaling behaviour, which is also demonstrated in the following simulation in Section 4.2.

3.2.2. Method of Stopping Time. As we know, an integervalued random variable $S<\infty$ is said to be a stopping time $[33,34]$ for the sequence $X_{0}, X_{1}, X_{2}, \ldots$, if the event $\{S=s\}$ is independent of $X_{s+1}, X_{s+2}, \ldots$, for all $s=0,1,2, \ldots$. The idea is that $X_{i}$ are observed one at a time: first $X_{0}$, then $X_{1}$, and so on; and $S$ represents the number observed when we stop. Notice that the above two first passage times, $T_{j}^{+}$and $T_{j}$, are stopping times associated with the PRW. After obtaining a spectra formula for the MFPT by the matrix formalism, we will use the stopping time technique to derive a probabilistic formula for the MFPT $\left\langle T_{i j}\right\rangle=E_{i} T_{j}^{+}$.

We now consider the PRW on the network, denoted by $\left(X_{t}: t=0,1,2, \ldots\right)$, which is a finite irreducible discrete Markov chain. Let $0<S<\infty$ be a stopping time such that $X_{S}=i$ and $E_{i} S<\infty$, and let $N_{j}(t)$ be the number of times the PRW visits node $j$ in $t$ steps. Viewing the PRW as the renewal process with the interrenewal time distribution $S$, from the reward-renewal theorem [33], one has

$$
\lim _{t \rightarrow \infty} \frac{N_{j}(t)}{t}=\frac{E_{i}\left(N_{j}(S)\right)}{E_{i} S}
$$

which, together with (2), leads to [44]

$$
E_{i}\left(N_{j}(S)\right)=\pi_{j} E_{i} S
$$

Next, we will show that many formulas of time scale related to the PRW are encoded in (32) and thus can be derived from (32) by particularly choosing $S$ and $j$. Further, we can combine these formulas to obtain the exact expression for MFPT $\left\langle T_{i j}\right\rangle=E_{i} T_{j}^{+}$. We would like to stress that this stopping time technique, including some formulas such as (38), (41), and (42) inferred by the technique, was proposed in [44]. We can also seek the sight of the method in the classical Markov theory [32, 34]. However, by using this method, we focus on the two aspects. On one hand, we use the method to get some new rigorous mathematical results for random walks on complex networks. On the other hand, we can apply this "probabilistic" approach to explore characteristics of dynamic processes in a randomwalk fashion such as random search, communication, and transportation in complex networks.

Taking $S=T_{i}^{+}$in (32), one has

$$
E_{i}\left(N_{j}\left(T_{i}^{+}\right)\right)=\pi_{j} E_{i} T_{i}^{+} .
$$

Setting $j=i$ gives

$$
E_{i}\left(N_{i}\left(T_{i}^{+}\right)\right)=1 \text {. }
$$

Using (33) and (34), we are led to an explicit expression for the MFRT to node $i$ as follows:

$$
\left\langle T_{i i}\right\rangle=E_{i} T_{i}^{+}=\frac{1}{\pi_{i}} .
$$

Introducing $S$ as "the first return to $i$ after the first visit to $j$," for $j \neq i$, one has

$$
E_{i}\left(N_{j}(S)\right)=E_{j}\left(N_{j}\left(T_{i}\right)\right)
$$

because there are no visits to $j$ before time $T_{j}$. Obviously,

$$
E_{i} S=E_{i} T_{j}+E_{j} T_{i}
$$

Substituting (36) and (37) into (32), we obtain the relation

$$
E_{j}\left(N_{j}\left(T_{i}\right)\right)=\pi_{j}\left(E_{j} T_{i}+E_{i} T_{j}\right) .
$$

Let us assume that the PRW starts out from node $i$ in the network. We fix a time $t_{0} \geq 1$ and set $S$ as the following 2stage stopping time: (i) wait time $t_{0}$ and then (ii) wait until the PRW next passages $i$ if necessary. Then (32), in the case where $j=i$, implies

$$
\pi_{i}\left(t_{0}+E_{\rho} T_{i}\right)=E_{i}\left(N_{i}(S)\right)=\sum_{t=0}^{t_{0}-1} p_{i i}^{(t)},
$$

where $\rho(\cdot)=P_{i}\left(X_{t_{0}}=\cdot\right)$.

Therefore,

$$
\sum_{t=0}^{t_{0}-1}\left(p_{i i}^{(t)}-\pi_{i}\right)=\pi_{i} E_{\rho} T_{i} .
$$

Considering (40) in the limit $t_{0} \rightarrow \infty$, we can write

$$
R_{i i} \equiv \sum_{t=0}^{\infty}\left(p_{i i}^{(t)}-\pi_{i}\right)=\pi_{i} E_{\pi} T_{i}
$$

where (3), that is, $\rho \rightarrow \pi\left(t_{0} \rightarrow \infty\right)$, was used.

In a similar way, with some calculation one obtains

$$
E_{k} T_{i}=\frac{1}{\pi_{i}}\left(R_{i i}-R_{k i}\right)
$$

Finally, combining (42), (35), and (10) yields our another central result, which can be summarized as follows.

For the PRW on the finite network, the MFPT of node $j$ from node $i$ is

$$
\left\langle T_{i j}\right\rangle= \begin{cases}\frac{1}{\pi_{j}}=\frac{\sum_{j} \sum_{i \in \tau(j)}\left(d_{i} d_{j}\right)}{\sum_{i \in \tau(j)}\left(d_{i} d_{j}\right)}, & i=j, \\ \frac{1}{\pi_{j}}\left(R_{j j}-R_{i j}\right), & i \neq j,\end{cases}
$$

where

$$
R_{i j}=\sum_{t=0}^{\infty}\left(p_{i j}^{(t)}-\pi_{j}\right)
$$


consequently, the AMFPT between all node pairs $i j(i \neq j)$ is

$$
\left\langle T_{i j}\right\rangle=\sum_{i} \sum_{j} \pi_{i} \pi_{j}\left\langle T_{i j}\right\rangle=\sum_{j} \pi_{j}\left\langle T_{i j}\right\rangle=\sum_{j} R_{j j},
$$

since $\sum_{j} R_{i j}=0$ for all $i \neq j$.

For the SRW on the finite network, by using the Laplace transform, the authors got similar theoretical result of MFPT in [11], given by

$$
\left\langle T_{i j}\right\rangle= \begin{cases}\frac{1}{\pi_{j}}=\frac{2 M}{d_{j}}, & i=j, \\ \frac{2 M}{d_{j}}\left(R_{j j}-R_{i j}\right), & i \neq j,\end{cases}
$$

where

$$
R_{i j}=\sum_{t=0}^{\infty}\left(p_{i j}^{(t)}-\pi_{j}\right)
$$

Remark 4. Compared with their method, the method here, that is, the stopping time technique, may be more "probabilistic." In fact, their result of (46) can also be obtained by this method. The key of the method lies in properly choosing the stopping time $S$ in (32), which seems to be a little tricky. It is worth noting that a special selection of $S$ can derive many other characteristic parameters. The MFPT in (43) or (46) is just one example. Thus, the stopping time technique may provide a powerful tool for understanding the scaling behaviour of random walks on complex networks.

From (43) or (46), it is easy to get the following relation.

$$
R_{j j}-R_{i j}=\frac{\left\langle T_{i j}\right\rangle}{\left\langle T_{j j}\right\rangle}, \quad i \neq j .
$$

From (43) and (46), the following equation can be got straightforwardly. Considering the SRW and PRW on the same finite network, if the node $j$ satisfies

$$
\sum_{i \in \tau(j)} d_{i}=\frac{\sum_{j} \sum_{i \in \tau(j)}\left(d_{i} d_{j}\right)}{2 M},
$$

then the mean first return times $\left\langle T_{j j}\right\rangle$ s of SRW and PRW starting from $j$ are equal.

Remark 5. As (43) and (46) show, the MFRT $\left\langle T_{j j}\right\rangle$ of SRW on the network is determined by the starting node's degree and inversely proportional to it, while the one of PRW starting out from node $j$ is determined by $\mathrm{CPD}_{j}$ and inversely proportional to $\mathrm{CPD}_{j}$. The MFPT $\left\langle T_{i j}\right\rangle$ of SRW on the network mainly depends on the degree of target node $j$, while the one of PRW mainly depends on $\mathrm{CPD}_{j}$. Simulations confirm analytical predictions and deepen discussions in Section 4.

Remark 6. In (43)-(47), $R_{i j}$ is an important quantity closely related to the mixing time of random walk [12]. The quantity depends on the network structure and the type of randomwalk strategy. Given that the mixing time is $T_{\text {mix }}, \sum_{t=0}^{T_{\operatorname{mix}}}\left(p_{i j}^{(t)}-\right.$ $\pi_{j}$ ) can be used as the approximation of $R_{i j}$. From the numerical results of random walks on NW small-world networks (and BA scale-free networks) presented in Figure 2 (and Figure 3 ) and according to (48), we find that the value $\left(R_{j j}-R_{i j}\right)$ is greater than 1 but very close to 1 .

Remark 7. From (30) and (45), the average over MFPTs from an arbitrary node to all other target nodes is identical to the AMFPT $\left\langle T_{i j}\right\rangle$ between all node pairs, where node $i$ or node $j$ is randomly chosen from all nodes according to the stationary distribution. This implies the average over MFPTs from a source node to all possible target nodes is not sensitively affected by the source node's location; numerical results are shown in Section 4.2.1.

\section{Simulations and Applications}

In this section, we make use of numerical simulation to deepen our discussions as well as confirm analytic results. In Section 4.1, based on theoretical results of (43), (46), and (29), we numerically explore the scaling properties of MFPT. Firstly, we use a simple random network to test the first passage property of the PRW. Secondly, we reveal topological properties of the NW small-world network such as assortativity and homogeneity through PRW and SRW. Then, through the comparison of PRW and SRW on the BA scale-free network, we investigate how the heterogeneous structure affects the scaling of MFPT. We also observe that PRW searches for the relatively high-degree node more quickly than SRW. In Section 4.2, based on theoretical results of (30) and (45), we numerically investigate the scaling behaviours of AMFPT. We find that the average over MFPTs from an arbitrary node to all other target nodes is identical to the AMFPT. We discuss the effects of the structural heterogeneity/homogeneity on the scaling of AMFPT. Further, we observe that for random walks on the BA scale-free network the AMFPT demonstrates approximatively linear scaling with the node number, that is, $\left\langle\left\langle T_{i j}\right\rangle\right\rangle \sim N$, and does not have the small-world feature, although the average shortest path length of the network has the small-world effect. This phenomenon also appears in the NW small-world network. The observation, to some extent, characterizes the network searchability [46]. Finally, we compare the scaling behaviours of average search steps among SRW, PRW, and maximum-degree-search (MDS) strategy. We explain why the scaling behaviours of average search steps for PRW and SRW are much similar, while being utterly different from the one for MDS.

\subsection{Scaling Properties of MFPT}

4.1.1. PRW on a Simple Random Network. The small connected random network is defined as $N=21$ labelled nodes and every pair of the nodes being connected with probability $p=0.1$ by using the ER model [1]. The average degree of the simple random network is $58 / 21$; namely, $\langle d\rangle=58 / 21$. 
TABLE 1: Simulation values and theoretical values of MFRT for PRW.

\begin{tabular}{lccccc}
\hline $\mathrm{CPD}_{j}$ & 72 & 90 & 100 & 105 & 132 \\
\hline Analytical $\left\langle T_{j j}\right\rangle$ & 10.778 & 8.622 & 7.76 & 7.390 & 5.878 \\
Numerical $\left\langle T_{j j}\right\rangle$ & 10.676 & 8.745 & 7.797 & 7.396 & 5.949 \\
\hline
\end{tabular}

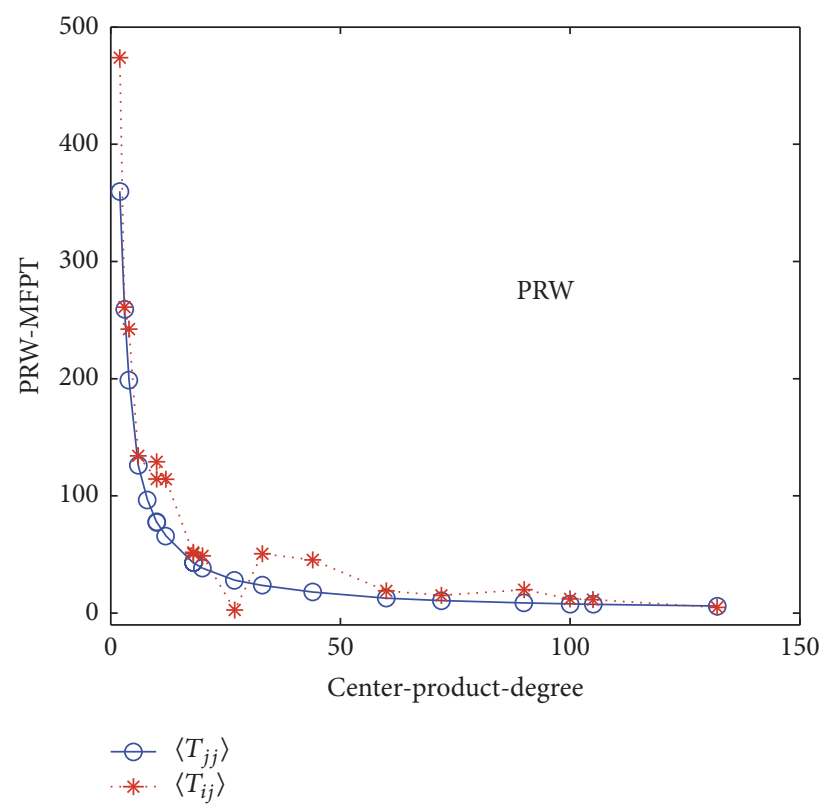

FIGURE 1: $\left\langle T_{j j}\right\rangle$ and $\left\langle T_{i j}\right\rangle$ versus node's center-product-degree $\mathrm{CPD}_{j}$ for PRW on the simple random network [source marked as $i=16$ and its degree $\left.d_{i}=1\right]$.

We perform PRW on the simple random network. Numerical data presented in the figures have been averaged over $10^{4}$ runs.

We perform PRW on the simple network; see Figure 1. For several nodes arbitrarily selected, both the analytical and numerical results presented in Table 1 claim that $\left\langle T_{j j}\right\rangle=$ $\sum_{j} \sum_{i \in \tau(j)}\left(d_{i} d_{j}\right) / \sum_{i \in \tau(j)}\left(d_{i} d_{j}\right)$. As is shown in Figure 1, our simulation states the MFPT $\left\langle T_{i j}\right\rangle$ of PRW on networks mainly depends on and is almost inversely proportional to the target node's center-product-degree, that is, $\left\langle T_{i j}\right\rangle \propto 1 / d_{j} \sum_{i \in \tau(j)} d_{i}$, which is also found in (43). That is, the simulation values of $\left\langle T_{i j}\right\rangle$ are in good agreement with theoretical predictions.

4.1.2. PRW on Small-World Networks: Comparison with $S R W$. The small-world network is generated by the method introduced by Newman [2]. In this network model, no edges are rewired, which is different from the small-world model proposed by Watts and Strogatz [3]. Instead shortcuts joining randomly chosen node pairs are added to the lowdimensional lattice. The size of the network is $N=100$, the neighboring number is $2 K=4$, and the probability of shortcuts is $p=0.15$. The average degree of the generated small-world network is $\langle d\rangle=18$. We perform PRW and SRW on the small-world network, respectively. Numerical data presented in the figures have been averaged over $10^{4}$ runs.

As shown in Figures 2(a) and 2(c), for SRW on the NW small-world network, the MFRT $\left\langle T_{j j}\right\rangle$ is determined by node's degree $d_{j}$, while for PRW, $\left\langle T_{j j}\right\rangle$ is determined by node's center-product-degree $\mathrm{CPD}_{j}$. Similar observation happens to the MFPT $\left\langle T_{i j}\right\rangle$ due to the fact that the value $\left(R_{j j}-R_{i j}\right)$ is greater than 1 but very close to 1 . In detail, for SRW on the NW small-world network, such value can be got from (48) and numerical results presented in Figures 2(a) and 2(b), while, for PRW, the value can be obtained from (48) and Figure 2(c). This further confirms the conclusions of (43) and (46) and improves the understanding of them.

(A) CPD-Based Assortativity. It is worth noticing that the random walks can be applied to reveal many different properties of network topology. Here is an example. One may be pleasantly surprised that $\left\langle T_{j j}\right\rangle$ and $\left\langle T_{i j}\right\rangle$ for SRW are determined by target node's degree $d_{j}$ as well as for PRW; see Figures 2(a) and 2(b). In fact, from Figure 2(c) or the analytic prediction of (43), $\left\langle T_{j j}\right\rangle$ and $\left\langle T_{i j}\right\rangle$ of PRW are mainly determined by and almost inversely proportional to $\mathrm{CPD}_{j}$. Hence, this observation indicates that the centerproduct-degree $\mathrm{CPD}_{j}$ of one node and the degree $d_{j}$ maintain consistency. That is, $\mathrm{CPD}_{j}$ of one node is an increasing function of $d_{j}$ for the NW small-world network. Thus, the NW small-world network considered here is weakly assortative, which, to a certain extent, reflects a homogeneous structure of the network.

(B) Search Efficiency. We compare SRW and PRW simultaneously on the same NW small-world network; see Figures 2(a) and 2(b). When the node's degree $d_{j}$ is sufficiently high, all $\left\langle T_{j j}\right\rangle_{\mathrm{s}}$ and $\left\langle T_{i j}\right\rangle_{\mathrm{s}}$ of PRW are smaller than those of SRW. It implies that PRW prefers the node with higher degree, which is in accordance with the analytical prediction of (49). This has some practical meanings. If PRW and SRW, as search processes on networks, search for target node with sufficiently high degree, the search time (walking steps) of PRW is much less than that of SRW. That is, in this case PRW searches more quickly and more efficiently.

4.1.3. PRW on Scale-Free Networks: Comparison with SRW. The scale-free network is generated by using BA model [4], with $m=2$ and network size $N=300$. The scale-free network generated in such a way has an exponent $\gamma=3$ of which the average degree is 4; namely, $\langle d\rangle=4$. We perform PRW and SRW on the scale-free network, respectively. Numerical data presented in the figures have been averaged over $10^{4}$ runs

(A) The Impacts of Structural Heterogeneity on the Scaling of $M F P T$. For SRW on the BA scale-free network, $\left\langle T_{j j}\right\rangle$ and $\left\langle T_{i j}\right\rangle$ are mainly determined by node's degree, whereas for PRW the two quantities fluctuate with node's degree; see Figures 3(a) and 3(b). Combining with Figure 3(c), it is easily seen that node's degree and center-product-degree in the BA scale-free network do not maintain consistency. This is entirely different from the above NW small-world network; see Figure 2. The difference is mainly because the degree distribution of the 


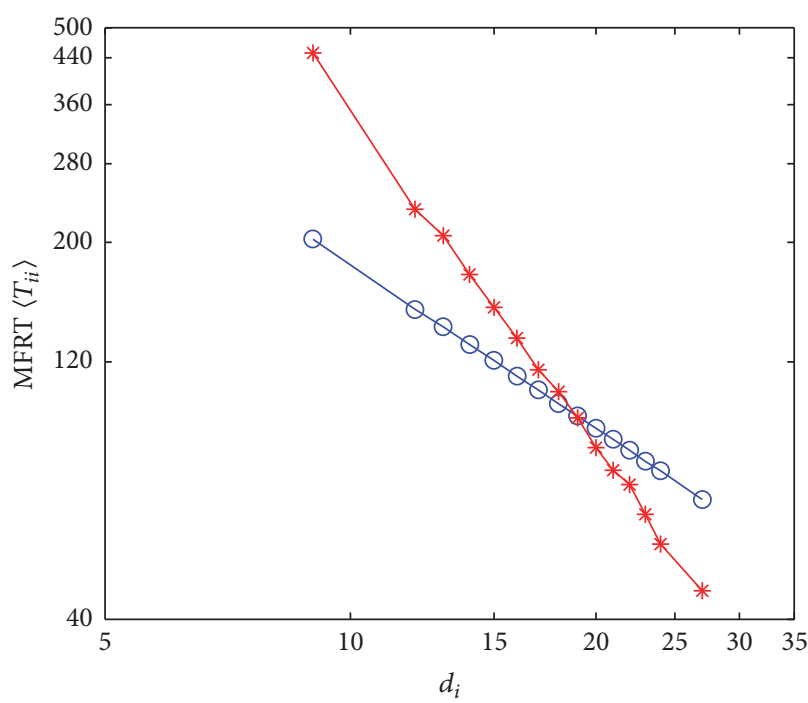

○ SRW

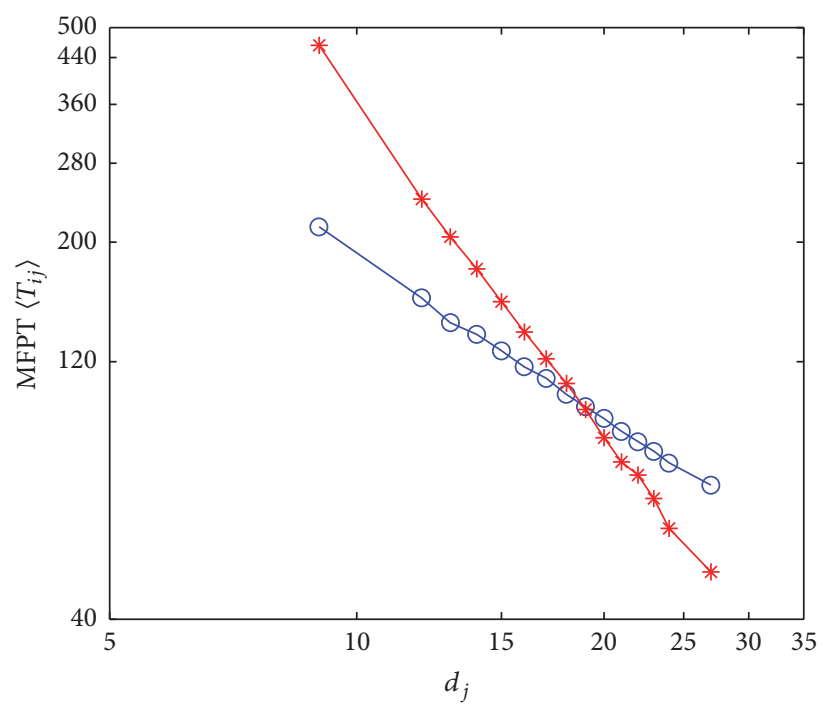

○ SRW

* PRW

(a)

(b)

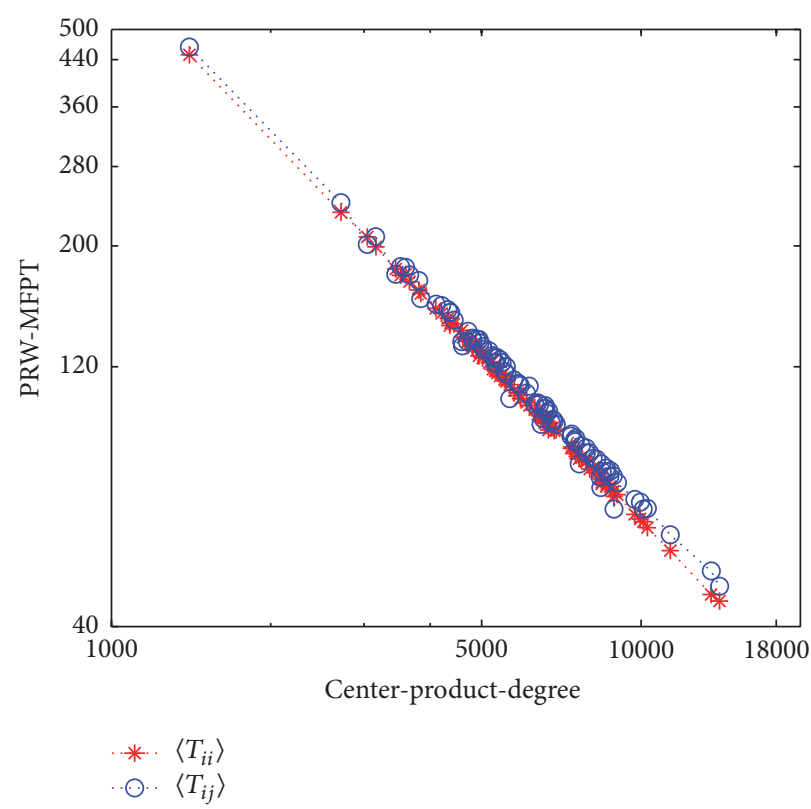

(c)

FIgURE 2: (Log-Log plots for random walks on NW small-world networks) (a) MFRT $\left\langle T_{i i}\right\rangle$ versus node's degree $d_{i}$. (b) MFPT $\left\langle T_{i j}\right\rangle$ versus target node's degree $d_{j}$. (c) MFRT of PRW $\left\langle T_{i i}\right\rangle$ versus node's center-product-degree $\mathrm{CPD}_{i}$; MFPT of PRW $\left\langle T_{i j}\right\rangle$ versus target node's centerproduct-degree $\mathrm{CPD}_{j}$ [source marked as $i=18$ and its degree $d_{i}=13$ ].

BA network has a power-law tail, or the BA network has a structure of heterogeneity. In fact, the weight allocation could play an important role in random-walk dynamics. The above observation also suggests that one can control the scaling behaviour of the random walks by configuring the edge weights. It should be emphasized that $\left\langle T_{j j}\right\rangle$ and $\left\langle T_{i j}\right\rangle$ of PRW mainly depend on the node's center-product-degree $\mathrm{CPD}_{j}$ rather than degree $d_{j}$. The discussion here may improve the understanding of the result in [24], where the authors studied biased random walks in uncorrelated networks and only explored the impacts of node's degree on the MFPT.

(B) Target Problem on Scale-Free Networks. Considering SRW and PRW as search strategies on networks, PRW prefers the high-degree node, while SRW searches for the relatively low-degree node more efficiently; see Figures 3(a) and 3(b). Since the scale-free network has a heterogeneous structure evidenced by the power-law degree distribution, this inspires 


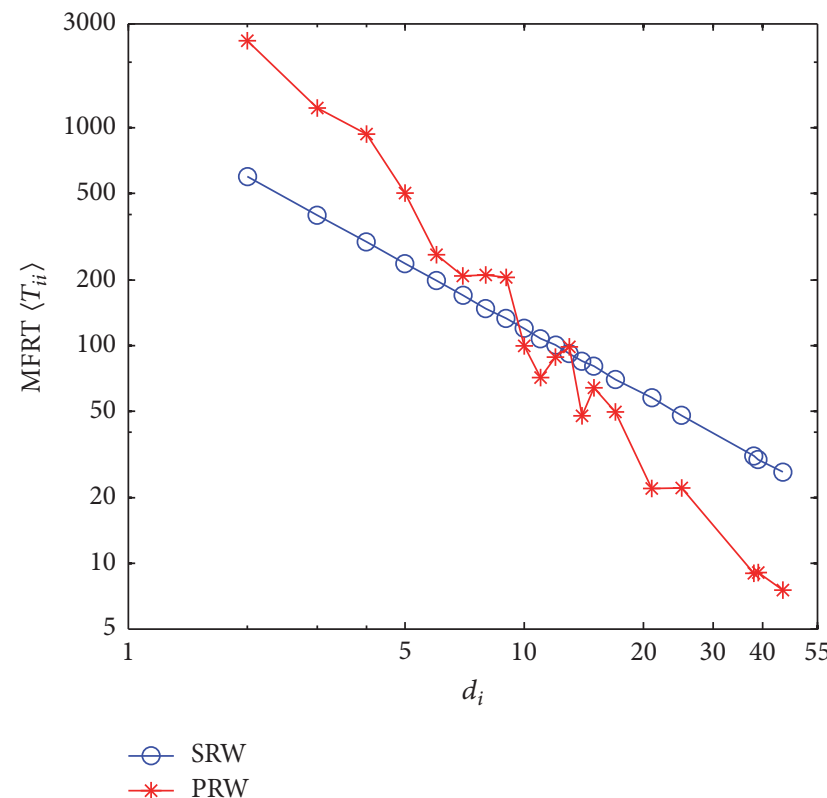

(a)

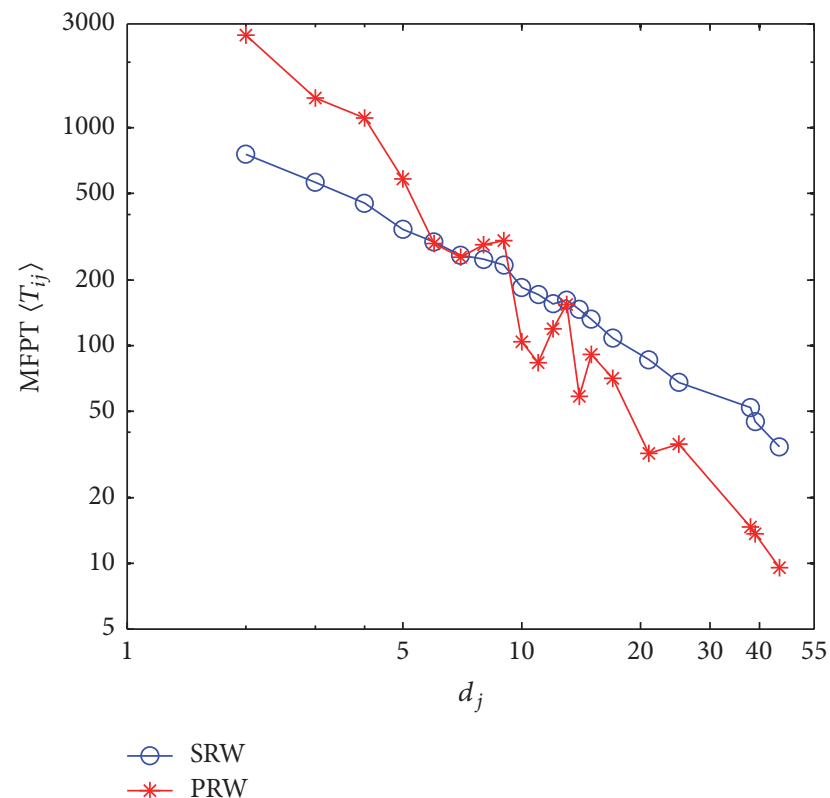

(b)

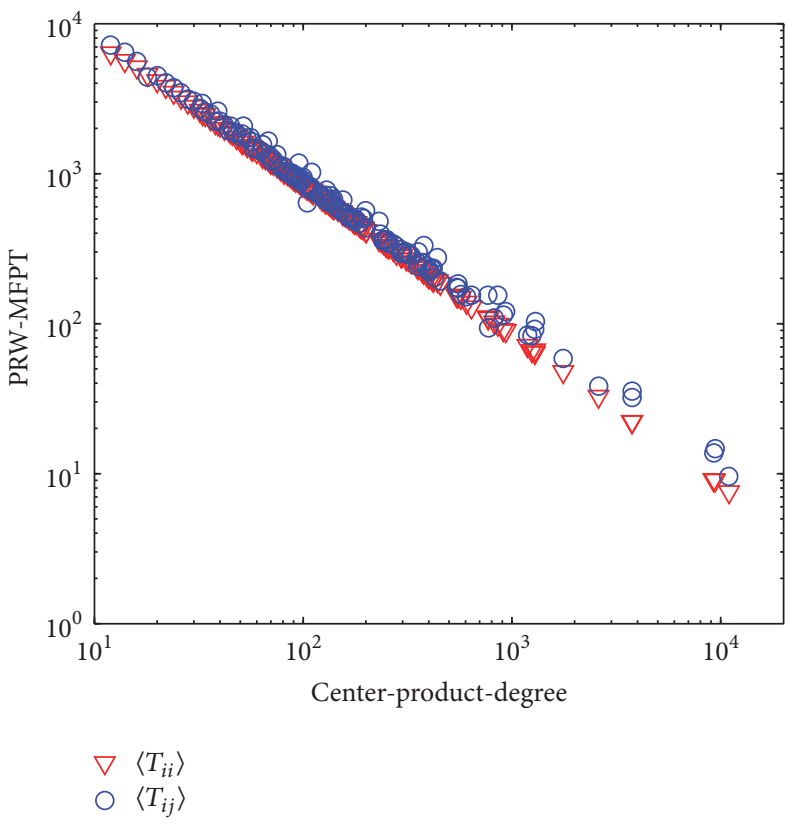

(c)

FIgURE 3: (Log-Log plots for random walks on BA scale-free networks) (a) MFRT $\left\langle T_{i i}\right\rangle$ versus node's degree $d_{i}$. (b) MFPT $\left\langle T_{i j}\right\rangle$ versus target node's degree $d_{j}$. (c) MFRT of PRW $\left\langle T_{i i}\right\rangle$ versus node's center-product-degree $\mathrm{CPD}_{i}$; MFPT of PRW $\left\langle T_{i j}\right\rangle$ versus target node's center-productdegree $\mathrm{CPD}_{j}$ [source marked as $i=146$ and its degree $d_{i}=3$ ].

us to propose a mixing navigation mechanism for search in scale-free networks, which interpolates between SRW and PRW. That is, to design the search strategy from source node to target node, one can firstly compare the size between the two nodes' degrees. If the target's degree is significantly higher than the source's degree or both of them are relatively high, then one could use PRW to search; otherwise one could use SRW alternatively.
4.2. Scaling Behaviours of AMFPT. In this part, the paradigmatic network models used are the same as those in Section 4.1. Based on the above discussion, we further numerically investigate scaling behaviours of AMFPT.

4.2.1. Sensitivity of the Total Average Search Cost Affected by the Source Node's Location. From Figure 4, one can see that the theoretical prediction of (30) and (45) agrees quite well 


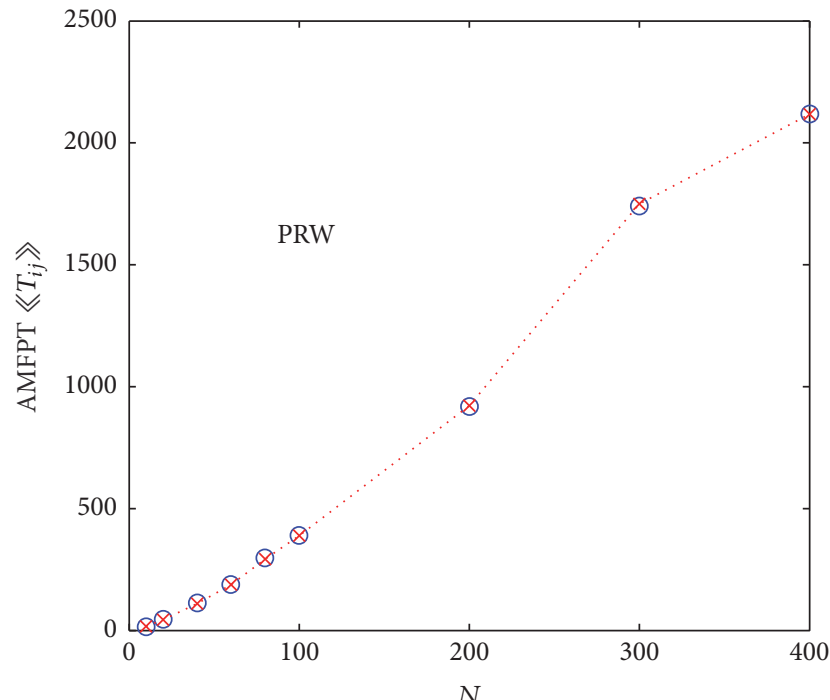

O MMFPT (from any one node) **. AMFPT $\left\langle T_{i j} 》\right.$

(a)

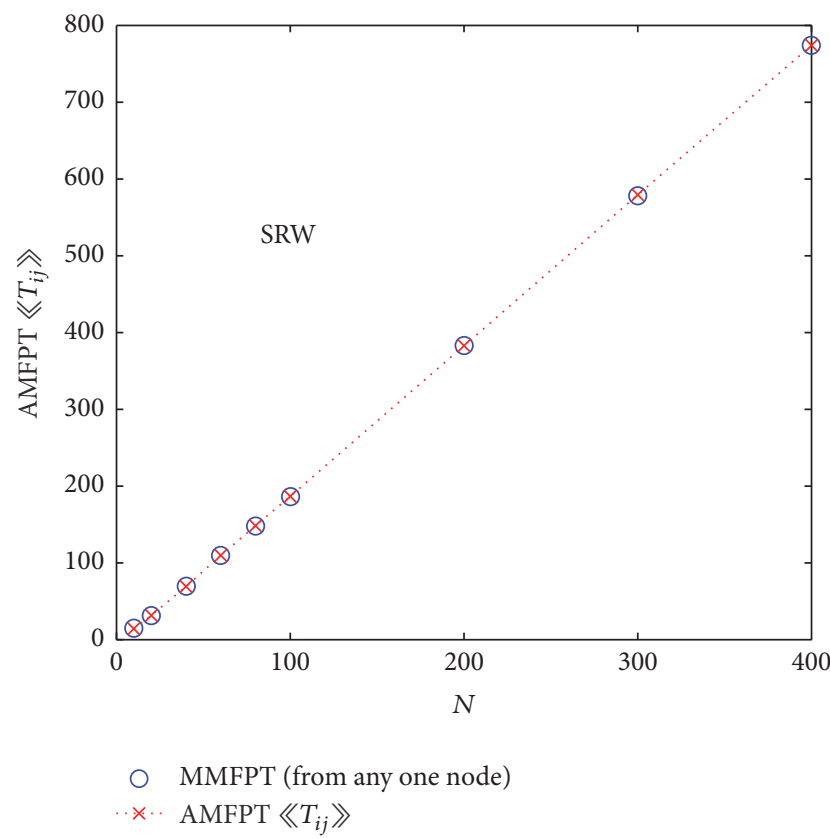

(b)

FIGURE 4: (a) PRW. (b) SRW. For random walks on the BA scale-free network, the AMFPT $\left\langle T_{i j} 》[\right.$ marked as $\times$ ] is equal to the average over MFPTs from one randomly chosen node $i$ to all other $N-1$ nodes [marked as o]. This is in accordance with the analytic result of (30) and (45).

with the numerical calculations. As expected, for PRW or SRW on different types of networks with different sizes $N$, for example, the BA scale-free network, the average MFPT from any one source node to all other destination nodes is equal to the AMFPT between all node pairs. Similar result was obtained for Koch networks [27]. Such result is interesting and one could still look into its further meaning. On one hand, this implies the average of MFPTs from a source node to all other destination nodes is not sensitively affected by the source node's location. On the other hand, if the PRW and SRW are two kinds of routing processes on scale-free networks, the total average search cost could be calculated by averaging from one site selected at random.

4.2.2. The Effects of Structural Heterogeneity on the Scaling of AMFPT. For the BA scale-free network with size $N$, the AMFPT $\left\langle T_{i j}\right\rangle$ of PRW is much greater than that of SRW; see Figure 5(a). That is, considering PRW and SRW as search strategies, the total average search cost of PRW is significantly higher than that of SRW. This is due to two reasons. One is that, compared with SRW, PRW tends to searching for the high-degree node; the other is that the degree distribution of the BA scale-free network is approximated by a power-law distribution; that is, the network has a heterogeneous structure. A few nodes have a large number of connections while most nodes have only a few connections. Thus, although PRW searching for high-degree nodes has high efficiency, to search for other nodes, PRW is prone to falling into the high-degreenode trap and difficultly reaches those nodes with only a few connections. All of these lead to the occurrence of the above phenomenon. Meanwhile, for the NW small-world network, there is little difference in the AMFPT $\left\langle T_{i j}\right\rangle$ between PRW and SRW, which is due to the fact that the degree distribution of the network is approximately Poisson distribution; that is, the network has a homogeneous structure; see Figure 5(b).

4.2.3. Network Searchability. As the actual average searching path length, the AMFPT $\left\langle T_{i j}\right\rangle$ of the random walk on the network can be regarded as one generalization of the average shortest path length, which, to some extent, characterizes network searchability [46]. The average shortest path length $\bar{L}$ of the BA network considered here obeys $\bar{L} \sim \log N$. The AMFPT $\left\langle T_{i j}\right\rangle$, however, satisfies $\left\langle T_{i j}\right\rangle \sim N$ for either PRW or SRW on the BA network; see Figure 5(a). Similar phenomenon happens to the NW network; see Figure 5(b). That is, in either case the actual average searching path length, that is, the AMFPT $\left\langle\left\langle T_{i j}\right\rangle\right.$, does not have the small-world effect. In short, the fact that a network has the small-world effect does not necessarily guarantee that it can be rapidly searched for.

4.2.4. The Difference of Scaling Behaviours for Search Cost among MDS, $P R W$, and $S R W$. The numerical result presented in Figure 5(a) shows that the AMFPT $\left\langle T_{i j}\right\rangle$ satisfies $\left\langle T_{i j}\right\rangle \sim N$ for either PRW or SRW on the BA network. The observation states that the leading scaling behaviours of $\left\langle\left\langle T_{i j}\right\rangle\right.$ between PRW and SRW are much similar, which is in accordance with the analytical result of (30). Incidentally, the conclusion just solves the authors' puzzlement in [18]. They applied MDS strategy to path finding in one scalefree network. In the MDS strategy, the neighbor node with the largest degree is tried first. Their results showed that 


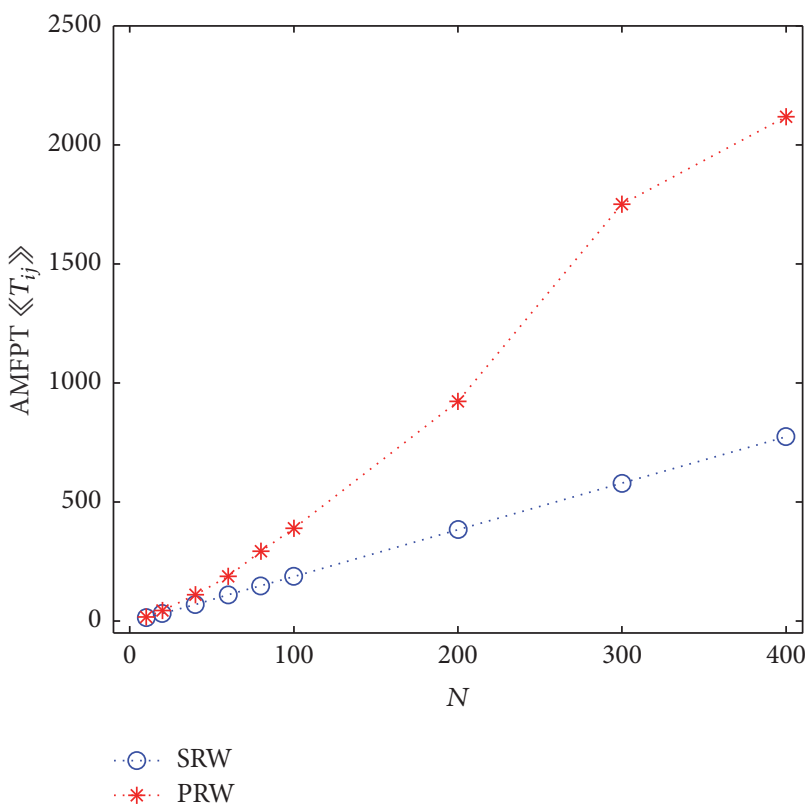

(a)

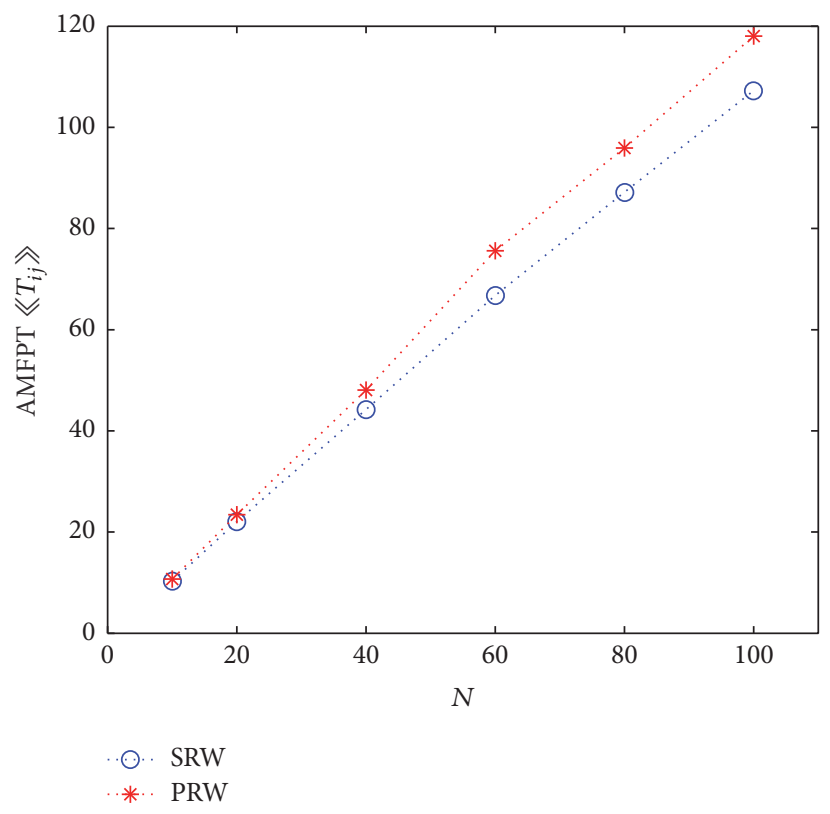

(b)

Figure 5: (a) AMFPT $\left\langle T_{i j}\right\rangle$ versus network size $N$ for PRW and SRW on the BA scale-free network. (b) AMFPT $《 T_{i j} 》$ versus network size $N$ for PRW and SRW on the NW small-world network. For the BA scale-free network, the gap in the AMFPT 《TT $\left.T_{i j}\right\rangle$ between PRW and SRW is more obvious than for the NW small-world network. Moreover, the fitting approximatively displays that $\left\langle T_{i j}\right\rangle \sim N$ holds for PRW or SRW on the two networks, respectively.

the global average search steps of MDS present small-world feature, $L_{\text {MDS }} \sim \log N$. They were puzzled by the fact that PRW and MDS strategies show very different scaling behaviours although both look quite similar, while PRW and SRW strategies demonstrate similar scaling behaviour. The reason for the fact is described as follows. The corresponding transition matrices of PRW and SRW are stochastic matrices and have similar spectral property [45], which implies $\left\langle\left\langle T_{i j}\right\rangle\right\rangle s$ of the two walks having the similar scaling behaviour due to (30). On the other hand, the PRW is one probabilistic degree-preferred mechanism, while the MDS is one deterministic degree-preferred strategy and the SRW is a uniform mechanism. The PRW incorporates the local degreepreferred element and the randomness ingredient, which in this sense can be regarded as a mixing strategy of SRW and MDS. Thus, their puzzlement just highlights the fact that, for PRW on the BA networks, the leading scaling behaviour of AMFPT is dominated by the randomness ingredient of the PRW.

\section{Conclusion}

In summary, we have developed a unified approach for understanding the scaling properties of discrete-time random walks on complex networks. Our work may be of practical significance for performing efficient search on complex networks and controlling the scaling behaviour of random walks on real-world networks.

We presented a systematic study of PRW in general undirected networks, including complex networks. We also made comparative study of PRW and SRW in order to better uncover and utilize the network structure. According to random walks on weighted networks, we attach weight $c_{i j}=$ $d_{j} d_{i}$ to each edge $(i, j)$, where $d_{i}$ and $d_{j}$ are the degrees of $i$ and $j$, and then construct PRW, of which the transition probability from node $i$ to node $j$ is proportional to the edge weight. We derived two exact expressions for the MFPT between two nodes, one of which is a spectra formula and the other is a probabilistic formula [see (29) and (43)]. We got explicitly the MFPT's dependence on the eigenvalues and eigenvectors of a matrix associated with the transition matrix of the PRW [see (29)]. We found that the CPD plays a main role in determining the scaling of MFPT for the PRW [see (43)]. The CPD of node $j$, being one measure of node strength, is defined as $d_{j} \sum_{i \in \tau(j)} d_{i}$, where $\tau(j)$ denotes all the connected neighbors of node $j$. Accordingly, we obtained the closed-form formulas of AMFPT between all node pairs and observed that the average over MFPTs from an arbitrary node to all other target nodes is equal to the AMFPT [see (30) and (45)].

Based on theoretical analysis, we did extensive simulations to confirm analytical predictions and deepen discussions. Through the comparison of PRW and SRW in networks, we revealed the CPD-based assortativity of network structure and found that the structural heterogeneity/homogeneity has a considerable impact on the scaling of MFPT and AMFPT. If we consider various random walks as search strategies applied to target problems, the MPFT between source and target characterizes search efficiency. The AMFPT represents the total average search cost, which, 
to some extent, can describe network searchability. We demonstrated that PRW prefers the high-degree node while SRW searches for the low-degree node more efficiently. We also found that the average over MFPTs from a source node to all possible destinations is not sensitively affected by the source node's location. As we observed, the average path length between nodes of a complex network possessing smallworld effect does not necessarily guarantee that one could perform search rapidly in the network. By comparing the search strategies of MDS, PRW, and SRW, we confirmed that the leading scaling behaviours of average search steps for PRW and SRW are much similar, while being utterly different from the one for MDS.

In the current work, we consider two paradigmatic types of single random walks on weighted network, that is, PRW and SRW corresponding to edge weights $c_{i j}=d_{j} d_{i}$ and $c_{i j}=1$. The generalization to more types of weight configurations would be interesting. Further, one could configure proper weights to develop proper multiple random walks for some practical applications such as improving the reliability and efficiency of searching for networks and identifying the influential nodes of real networks [47]. We leave these more intriguing problems to future studies.

\section{Conflicts of Interest}

The authors declare that there are no conflicts of interest regarding the publication of this paper.

\section{Acknowledgments}

This work is partially supported by Soft Science Research Project of Shanghai Songjiang Science and Technology Commission under Grant no. 17SJRKT40; Soft Science Research Project of Shanghai Science and Technology Commission under Grant no. 17692109000; National Natural Science Foundation of China, under Grant nos. 11471211, 11171018, and 61402281; and Shanghai Natural Science Fund Project under Grant no. 14ZR1418900.

\section{References}

[1] S. Boccaletti, V. Latora, Y. Moreno, M. Chavez, and D.-U. Hwang, "Complex networks: structure and dynamics," Physics Reports. A Review Section of Physics Letters, vol. 424, no. 4-5, pp. 175-308, 2006.

[2] M. E. Newman, "The structure and function of complex networks," SIAM Review, vol. 45, no. 2, pp. 167-256, 2003.

[3] D. J. Watts and S. H. Strogatz, "Collective dynamics of 'smallworld' networks," Nature, vol. 393, no. 6684, pp. 440-442, 1998.

[4] A.-L. Barabási and R. Albert, "Emergence of scaling in random networks," American Association for the Advancement of Science. Science, vol. 286, no. 5439, pp. 509-512, 1999.

[5] K. Li, Z. Ma, Z. Jia, M. Small, and X. Fu, "Interplay between collective behavior and spreading dynamics on complex networks," Chaos. An Interdisciplinary Journal of Nonlinear Science, vol. 22, no. 4, Article ID 043113, 043113, 10 pages, 2012.

[6] W.-X. Wang, B.-H. Wang, B. Hu, G. Yan, and Q. Ou, "General dynamics of topology and traffic on weighted technological networks," Physical Review Letters, vol. 94, no. 18, Article ID 188702, 2005.

[7] Y. Li, S. Tong, and T. Li, "Observer-based adaptive fuzzy tracking control of MIMO stochastic nonlinear systems with unknown control direction and unknown dead-zones," IEEE Transactions on Fuzzy Systems, vol. 23, no. 4, pp. 1228-1241, 2015.

[8] X. Zhao, P. Shi, X. Zheng, and L. Zhang, "Adaptive tracking control for switched stochastic nonlinear systems with unknown actuator dead-zone," Automatica. A Journal of IFAC, the International Federation of Automatic Control, vol. 60, pp. 193-200, 2015.

[9] Y. Li, S. Sui, and S. Tong, "Adaptive fuzzy control design for stochastic nonlinear switched systems with arbitrary switchings and unmodeled dynamics," IEEE Transactions on Cybernetics, vol. 47, no. 2, pp. 403-414, 2017.

[10] H. Wang, W. Sun, and P. X. Liu, "Adaptive intelligent control of nonaffine nonlinear time-delay systems with dynamic uncertainties," IEEE Transactions on Systems, Man, and Cybernetics: Systems, no. 99, pp. 1-12, 2016.

[11] J. D. O. Noh and H. Rieger, "Random walks on complex networks," Physical review letters, vol. 92, no. 11, p. 118701, 2004.

[12] R. Durrett, Random graph dynamics, Cambridge University Press, Cambridge, UK, 2007.

[13] S. A. Pandit and R. E. Amritkar, "Random spread on the family of small-world networks," Physical Review E, vol. 63, no. 4, 2001.

[14] S. Jespersen, I. M. Sokolov, and A. Blumen, "Relaxation properties of small-world networks," Physical Review E - Statistical Physics, Plasmas, Fluids, and Related Interdisciplinary Topics, vol. 62, no. 3 B, pp. 4405-4408, 2000.

[15] W. Sun, "Random walks on generalized Koch networks," Physica Scripta, vol. 88, no. 4, Article ID 045006, 2013.

[16] J. D. Noh and S. Kim, "Random-walk and pair-annihilation processes on scale-free networks," Journal of the Korean Physical Society, vol. 48, pp. 202-207, 2006.

[17] Z. Zheng, H. Wang, S. Gao, and G. Wang, "Comparison of multiple random walks strategies for searching networks," Mathematical Problems in Engineering, vol. 2013, Article ID 734630, 2013.

[18] B. J. Kim, C. N. Yoon, S. K. Han, and H. Jeong, "Path finding strategies in scale-free networks," Physical Review E - Statistical, Nonlinear, and Soft Matter Physics, vol. 65, no. 2, Article ID 027103, p. 027103/4, 2002.

[19] N. Perra, A. Baronchelli, D. Mocanu, B. Gonçalves, R. PastorSatorras, and A. Vespignani, "Random walks and search in time-varying networks," Physical Review Letters, vol. 109, no. 23, Article ID 238701, 2012.

[20] S.-P. Wang and W.-J. Pei, "Detecting unknown paths on complex networks through random walks," Physica A: Statistical Mechanics and its Applications, vol. 388, no. 4, pp. 514-522, 2009.

[21] H. Tian, H. Shen, and T. Matsuzawa, "Random walk routing for wireless sensor networks," in Proceedings of the 6th International Conference on Parallel and Distributed Computing, Applications and Technologies, PDCAT 2005, pp. 196-200, chn, December 2005.

[22] V. M. López Millán, V. Cholvi, L. López, and A. Fernández Anta, "A model of self-avoiding random walks for searching complex networks," Networks, vol. 60, no. 2, pp. 71-85, 2012.

[23] C.-M. Angelopoulos, S. Nikoletseas, D. Patroumpa, and J. Rolim, "Coverage-adaptive random walks for fast sensory data collection," Lecture Notes in Computer Science (including subseries Lecture Notes in Artificial Intelligence and Lecture Notes in Bioinformatics), vol. 6288, pp. 81-94, 2010. 
[24] A. Fronczak and P. Fronczak, "Biased random walks in complex networks: The role of local navigation rules," Physical Review E - Statistical, Nonlinear, and Soft Matter Physics, vol. 80, no. 1, Article ID 016107, 2009.

[25] S. Lee, S.-H. Yook, and Y. Kim, "Searching method through biased random walks on complex networks," Physical Review E - Statistical, Nonlinear, and Soft Matter Physics, vol. 80, no. 1, Article ID 017102, 2009.

[26] A. P. Riascos and J. L. Mateos, "Long-range navigation on complex networks using Lévy random walks," Physical Review E - Statistical, Nonlinear, and Soft Matter Physics, vol. 86, no. 5, Article ID 056110, 2012.

[27] Z. Zhang and S. Gao, "Scaling of mean first-passage time as efficiency measure of nodes sending information on scale-free Koch networks," European Physical Journal B, vol. 80, no. 2, pp. 209-216, 2011.

[28] S. Hwang, D.-S. Lee, and B. Kahng, "First passage time for random walks in heterogeneous networks," Physical Review Letters, vol. 109, no. 8, Article ID 088701, 2012.

[29] S. Condamin, O. Bénichou, V. Tejedor, R. Voituriez, and J. Klafter, "First-passage times in complex scale-invariant media," Nature, vol. 450, no. 7166, pp. 77-80, 2007.

[30] V. Tejedor, O. Bénichou, and R. Voituriez, "Close or connected: Distance and connectivity effects on transport in networks," Physical Review E - Statistical, Nonlinear, and Soft Matter Physics, vol. 83, no. 6, Article ID 066102, 2011.

[31] Y. Lin, A. Julaiti, and Z. Zhang, "Mean first-passage time for random walks in general graphs with a deep trap," Journal of Chemical Physics, vol. 137, no. 12, Article ID 124104, 2012.

[32] O. Häggström, Finite Markov Chains And Algorithmic Applications, Cambridge University Press, Cambridge, UK, 2002.

[33] S. M. Ross, Stochastic Processes, John Wiley, New York, NY, USA, 1996.

[34] R. Durrett, Probability: Theory and Examples, Cambridge University Press, Cambridge, UK, 4th edition, 2010.

[35] Z. T. Zheng, Exploring complex networks by random graph evolution and random walks [Ph.D. Dissertation], Shanghai University, Shanghai, China, 2009.

[36] Z. Zhang, T. Shan, and G. Chen, "Random walks on weighted networks," Physical Review E - Statistical, Nonlinear, and Soft Matter Physics, vol. 87, no. 1, Article ID 012112, 2013.

[37] A. Barrat, M. Barthélemy, R. Pastor-Satorras, and A. Vespignani, "The architecture of complex weighted networks," Proceedings of the National Academy of Sciences of the United States of America, vol. 101, no. 11, pp. 3747-3752, 2004.

[38] Y. Li, Z. Ma, and S. Tong, "Adaptive Fuzzy Output-Constrained Fault-Tolerant Control of Nonlinear Stochastic Large-Scale Systems With Actuator Faults," IEEE Transactions on Cybernetics, vol. 47, no. 9, pp. 2362-2376, 2017.

[39] H. Wang, P. X. Liu, and P. Shi, "Observer-based fuzzy adaptive output-feedback control of stochastic nonlinear multiple timedelay systems," IEEE Transactions on Cybernetics, vol. 47, no. 9, pp. 2568-2578, 2017.

[40] Y. M. Li and S. C. Tong, "Adaptive fuzzy output constrained control design for multi-input multioutput stochastic nonstrictfeedback nonlinear systems," IEEE Transactions on Cybernetics, no. 99, pp. 1-10, 2016.

[41] X. Zhao, P. Shi, X. Zheng, and J. Zhang, "Intelligent tracking control for a class of uncertain high-order nonlinear systems," IEEE Transactions on Neural Networks and Learning Systems, vol. 27, no. 9, pp. 1976-1982, 2016.
[42] L. Lovász, "Random walks on graphs: a survey," in Combinatorics, Paul Erdös is eighty, vol. 2, pp. 1-46, János Bolyai Mathematical Society, Budapest, Hungary, 1993.

[43] C. M. Grinstead and J. L. Snell, Introduction to Probability, American Mathematical Society, Providence, RI, USA, 1997.

[44] D. J. Aldous and J. Fill, "Reversible Markov Chains and Random Walks on Graphs, (monograph in preparation)," http://www.stat.berkeley.edu/ãldous/RWG/book.html, 2002.

[45] F. R. Chung, Spectral Graph Theory, American Mathematical Society, Providence, RI, USA, 1997.

[46] J. M. Kleinberg, "Navigation in a small world," Nature, vol. 406, no. 6798, p. 845, 2000.

[47] M. Kitsak, L. K. Gallos, S. Havlin et al., "Identification of influential spreaders in complex networks," Nature Physics, vol. 6, no. 11, pp. 888-893, 2010. 


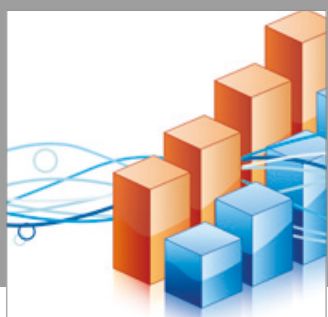

Advances in

Operations Research

vatersals

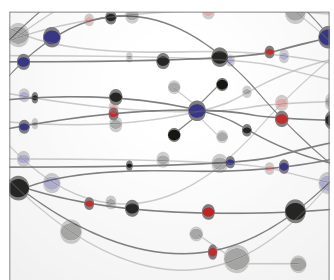

\section{The Scientific} World Journal
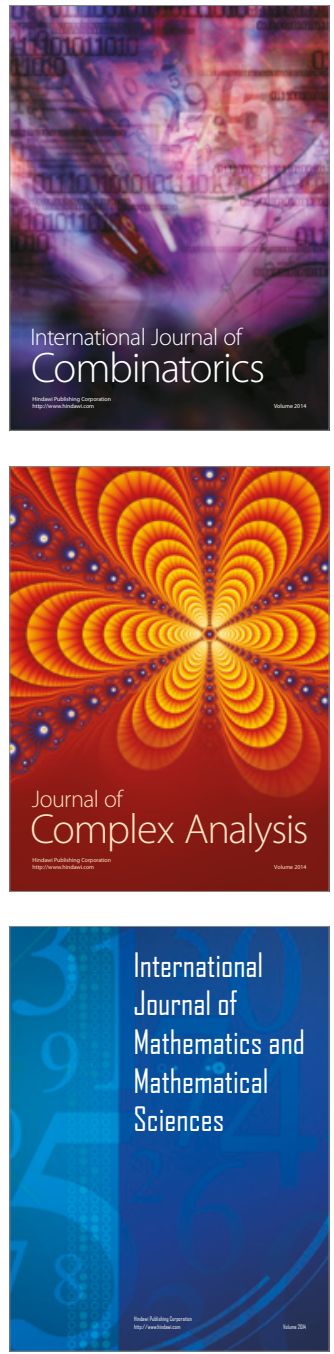
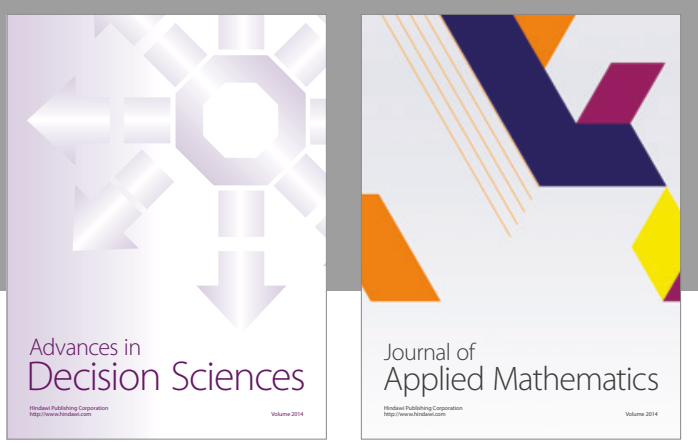

Algebra

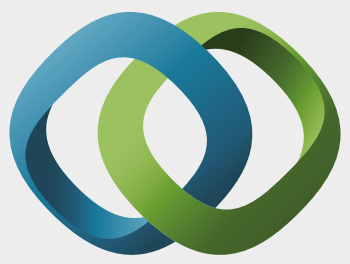

\section{Hindawi}

Submit your manuscripts at

https://www.hindawi.com
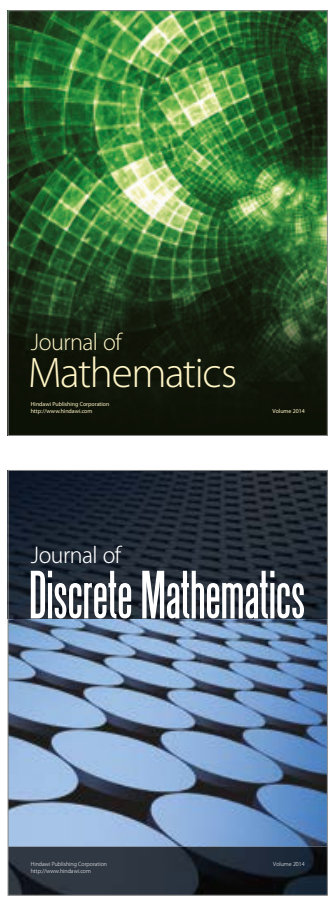

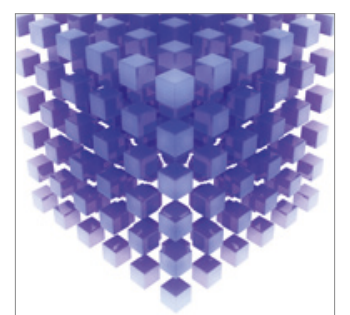

Mathematical Problems in Engineering
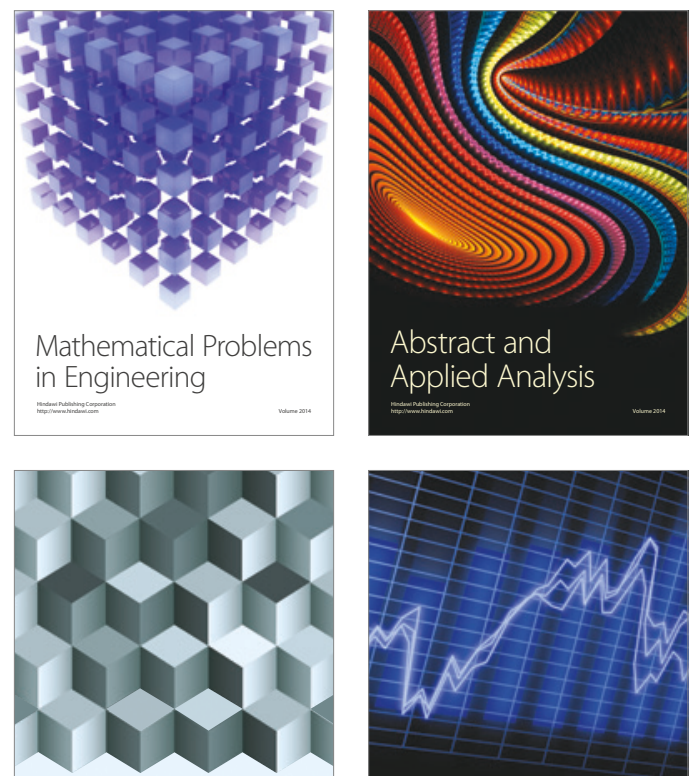

Journal of

Function Spaces

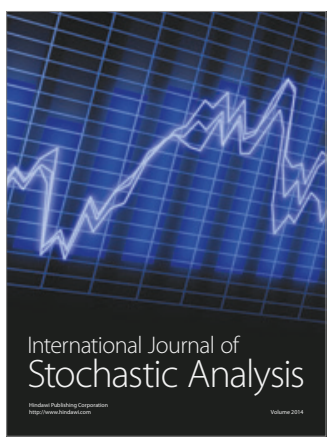

Probability and Statistics
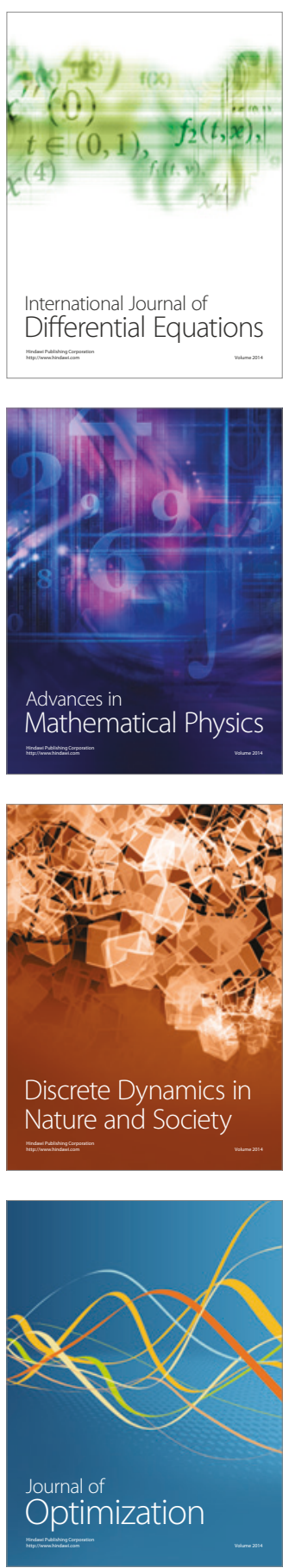